\title{
Commentaries
}

\section{Microsatellite instability in colitis associated colorectal cancer}

As molecular pathways of colon carcinogenesis continue to be defined for high risk hereditary colon cancer syndromes and for average risk sporadic colon cancers, it seems that colon carcinogenesis in the setting of chronic idiopathic inflammatory bowel disease (IBD) may be unique. ${ }^{1}$ Although to some extent this notion seems intuitive because of the substrate of chronic inflammation from which these cancers arise, it is nevertheless curious that despite the setting of chronic inflammation, colon cancers occurring in patients with colitis share several features in common with those that arise in patients with hereditary non-polyposis colorectal cancer (HNPCC). For example, in both conditions there is a tendency for the colorectal cancers to affect young individuals, be multifocal, show a proximal colonic distribution, and display mucinous, signet-ring cell or undifferentiated histology. In addition, HNPCC colorectal cancers often have a rim of surrounding inflammation, referred to as a Crohn's-like reaction. These clinicopathological similarities have prompted some investigators to explore whether colitis associated cancers might in fact share a common molecular pathogenesis with HNPCC colorectal cancers.

Colorectal cancers in patients with HNPCC occur by virtue of a defective ability to repair DNA base pair mismatches. This results in DNA replication errors that have the net effect of altering genes that are critical for maintaining normal growth and behaviour of colonic epithelial cells. Such replication errors can be identified using markers that detect short repetitive sequences, or microsatellites, located throughout the genome. Errors in replicating these sequences result in a phenotype termed microsatellite instability (MI). Several genes act in concert to orchestrate normal repair of DNA base mismatches, including hMLH1, hMSH2, hMSH3, hMSH6, hPMS1, and hPMS2. ${ }^{2}$ Except for hMSH3, germline mutations of any one of these genes are known to give rise to tumours of the colorectum and other organs in patients with HNPCC, with mutations of hMLH1 and hMSH2 accounting for the vast majority of known HNPCC families.

In this issue (see page 367), Cawkwell and colleagues investigated the frequency of MI in colitis associated colorectal cancers to determine whether these tumours might share a similar molecular pathogenesis with HNPCC. They analysed 46 colitis associated colonic adenocarcinomas for MI using a panel of four markers in a fluorescence PCR-based MI assay, and also stained tumours by using immunohistochemistry for loss of hMLH1 and hMSH2. Six $(15 \%)$ of 41 cases demonstrated $\mathrm{MI}$ at one or more marker, whereas only one $(2.4 \%)$ case had MI at two or more of the four markers. The latter case was also the only one that demonstrated loss of immunohistochemical staining of hMSH2, a useful surrogate marker of higher rates of $\mathrm{MI}$ in tumours. The authors suggest that $\mathrm{MI}$ is uncommon in colitis associated colon cancers, thus distinguishing these cancers from HNPCC where the frequency of MI positivity is about $85 \%$, but perhaps somewhat similar to sporadic colon cancers which have a $15 \%$ MI positivity rate.

Before accepting that $\mathrm{MI}$ is uncommon in colitis associated neoplasms, it is worth considering that other investigators have reported a somewhat higher frequency of $\mathrm{MI}$ in colitis associated dysplasias and cancers. Using a panel of five microsatellite markers, Suzuki et al noted MI of at least one marker in 17/63 (27\%) patients, and 15/120 $(8.3 \%)$ dysplastic and cancerous lesions manifested MI of two or more markers. ${ }^{3}$ These investigators also reported that some colitis associated neoplasms manifested mutations of transforming growth factor $\beta 1$ type II receptor, a target gene that is often affected by MI in HNPCC. ${ }^{4}$ Brentnall and coworkers used a panel of 13 markers and observed MI of at least one marker in $40 \%$ of cancers and $85 \%$ of high grade dysplasias, and $\mathrm{MI}$ of at least two markers in $40 \%$ of cancers and $46 \%$ of high grade dysplasias. ${ }^{5}$ In that study, MI was quite frequent even in colitic mucosa that was non-dysplastic, regardless of whether the patient had colitis of short (less than three years) or long (more than eight years) duration, and MI was not found in acute, self limited forms of colitis. Furthermore, Heinen and colleagues used a panel of six microsatellite markers and noted MI at one locus in 13\% non-dysplastic, 5\% dysplastic, and $17 \%$ cancerous lesions in colitis, and curiously, only non-dysplastic mucosa manifested MI at two loci. ${ }^{6}$

How can we reconcile the differences among these studies? To be sure, the studies differ with regard to the number and types of neoplastic tissues analysed and to some extent the method of analysing MI. However, the crucial difference relates to the choice and number of markers used in the various studies. Because MI testing has been so variable between laboratories, a National Cancer Institute workshop established consensus recommendations in 1998 suggesting that a reference panel of five markers be used to classify a tumour as having either a high (MI-H), low (MI$\mathrm{L})$, or stable (MS) microsatellite phenotype. ${ }^{7}$ If the recommended five loci are analysed, a tumour is classified as MI-L if one locus exhibits instability, or MI-H if two or more loci are unstable. If more than the five markers are used, MI-L status would apply to tumours manifesting instability at less than $30-40 \%$ of loci, whereas tumours with instability of more than $30-40 \%$ of loci would be considered MI-H. Importantly, included among the five reference markers are BAT25 and BAT26, which recognise mononucleotide repeats and are therefore considered more sensitive markers. It is possible that if Cawkwell and coworkers had used these more sensitive markers, the frequency of MI in their colitis associated cancers might in fact be higher than reported.

As we await additional studies using standard markers, a common theme seems to already be emerging from the existing literature: microsatellite instability, especially the MI-L phenotype, may in fact be fairly common in ulcerative colitis, both in neoplastic and non-neoplastic tissue. Some hypothesise that if the DNA repair machinery is defective in ulcerative colitis mucosa, the oxidative stress that accompanies chronic inflammation can damage DNA and overwhelm the ability of the colonocytes to repair DNA, resulting in the MI phenotype of even non-dysplastic mucosa. Although the biological and clinical significance of low level 
$\mathrm{MI}$ is not yet clear, this issue is receiving increasing attention. For example, germline hMSH6 mutations have recently been reported to give rise to an attenuated HNPCC phenotype. ${ }^{8}$ Moreover, based upon observations made in yeast models, inactivation or inhibition of hMSH6 might result in errors at mononucleotide tracts, but not dinucleotide or longer repeat elements. ${ }^{9}$ This raises the possibility that mutations of hMSH6 or perhaps silencing of the gene by methylation might contribute to the neoplastic process in ulcerative colitis, a prospect worthy of further investigation.

S H ITZKOWITZ

The Dr Burrill B Crohn Professor of Medicine,

Director, The Dr Henry D fanowitz Division of Gastroenterology, Box 1069, GI Division,

Mount Sinai School of Medicine,

One Gustave Levy Place,

New York, NY 10029, USA

email:sitzk@smtplink.mssm.edu
1 Itzkowitz SH. Inflammatory bowel disease and cancer. Gastroenterol Clin North Am 1997;26:129-39.

2 Kolodner RD, Marsischky GT. Eukaryotic DNA mismatch repair. Curr Opin Genet Dev 1999;9:89-96.

3 Suzuki H, Harpaz N, Tarmin L, et al. Microsatellite instability in ulcerative colitis-associated colorectal dysplasias and cancers. Cancer Res 1994;54: 4841-4

4 Souza RF, Lei J, Yin J, et al. A transforming growth factor $\beta 1$ receptor type II mutation in ulcerative colitis-associated neoplasms. Gastroenterology 1997;112:40-5.

5 Brentnall TA, Crispin DA, Bronner MP, et al. Microsatellite instability in nonneoplastic mucosa from patients with chronic ulcerative colitis. Cancer Res 1996;56:1237-40.

6 Heinen CD, Noffsinger AE, Belli J, et al. Regenerative lesions in ulcerative colitis are characterized by microsatellite mutation. Genes Chromosom Cancer 1997;19:170-5.

7 Boland CR, Thibodeau SN, Hamilton SR, et al. A National Cancer Institute workshop on microsatellite instability for cancer detection and familial predisposition: development of international criteria for the determination of microsatellite instability in colorectal cancer. Cancer Res 1998;58:5248-

8 Kolodner R, Tytell JD, Schmeits JL, et al. Germline msh6 mutations in colorectal cancer families. Cancer Res 1999;59:5068-74.

9 Marsischky GT, Filosi N, Kane MF, et al. Redundancy of Saccharomyces cerevisiae MSH3 and MSH6 in MSH2-dependent mismatch repair. Genes Dev 1996;10:407-20.

See article on page 395

\section{How should endoscopic accessories be selected: trial or error?}

Before being put on the market, medicines undergo stringent evaluation of their efficacy and safety, satisfying the regulatory bodies of individual countries. The costs of these activities are borne by manufacturers or developers who may later be held responsible for subsequent shortcomings. By contrast, medical devices, as for example those used in endoscopic procedures, require no such prior assessment in many countries. In the United Kingdom, since June 1998, all new products must satisfy the requirements of the Medical Devices Agency, ${ }^{1}$ the UK Competent Authority, to be awarded the $\mathrm{CE}$ mark allowing use throughout the European Community. Endoscopists and assistants commonly learn of new products from commercial stands at exhibitions held alongside scientific meetings or from manufacturers' representatives or sales staff. The apparent virtues and novelty of products are exhorted whereas objective clinical evidence of efficacy or safety is usually not available. Phase III development is not a clearly defined prerequisite before device marketing. Why should devices be less well investigated and evaluated than drugs when offered for sale? The initiative and ingenuity of manufacturers in developing and producing new equipment is to be warmly commended, but enthusiasm for sales ahead of rigorous clinical evaluation must be seriously questioned.

Endoscopists are confronted with an overwhelming range of equipment from competing companies and at present choices are based upon previous experience, recommendations from colleagues and an inspection of the product. This situation is unsatisfactory and devices must be subjected to the same rigorous evaluation as medicines. Who should organise such trials? Ideally, the manufacturers in necessary collaboration with clinicians, obeying the rules of controlled trials with ethical approval. The expense would be considerable but less than in the case of medicines. Moreover the time between development and commercial release would be similarly prolonged-but would this necessarily be a bad thing? Many products currently offered for sale would not reach the market place.

One alternative approach is for trials to be organised by clinicians, perhaps with commercial sponsorship, for independent publication in peer-reviewed journals. The paper by England et al in this issue (see page 395) is an excellent example of such collaboration, investigating two designs of stents for palliation of malignant obstruction of the bile duct. The study was supported by the equipment manufacturers and the findings were analysed by a professional monitoring group according to European Good Clinical Practice guidelines.

Bacterial colonisation and encrustation of plastic biliary stents has been shown by in vitro studies to be affected by the surface characteristics of the stent material and in in vivo studies by the structure of the stent used. Much interest has focused on the potential for reducing encrustation by removal of side holes from the stent design - for example, the "fir tree" or Tannenbaum stent. A previous non-randomised study showed significantly improved patency compared with the pig tail design. ${ }^{2}$ Patency rates for $12 \mathrm{~F}$ and $10 \mathrm{~F}$ straight stents are similar whereas $7 \mathrm{~F}$ and $8 \mathrm{~F}$ stents are more prone to occlusion. ${ }^{3-5}$ Use of plastic stents in the palliation of malignant obstructive jaundice has been challenged by the superior performance of large bore expanding metal stents with improved patency rates revealed in four prospective randomised trials. ${ }^{6-9}$ However, at detection, the majority of such strictures are caused by pancreatic malignancy often with a median survival less than the median patency of plastic stents. Many of those who develop recurrent jaundice are not restented with patients' wishes and compliance rather than procedural logistics proposed as the major factors. ${ }^{10}$ Diagnostic uncertainty at the outset reserves a role for an easily removable and exchangeable stent-factors inherent in plastic constructions. Whether the choice of stent affects overall survival is less clear.

England et al have looked at a remaining issue of whether the $10 \mathrm{~F}$ Teflon Tannenbaum stent or the more commonly used $10 \mathrm{~F}$ polyethylene Cotton-Leung stent with side holes, achieved longer patency. In a well planned prospective multicentre study they randomised at deep cannulation 134 patients presenting with a malignant common duct stricture to palliation using either the Tannenbaum or the CottonLeung design. All patients received prophylactic antibiotics at stenting. Respective groups of 65 and 69 patients were similar in age, type of malignancy (over $60 \%$ pancreatic), length of stricture, and American Society of Anesthesiologists' classification. There was an overall generous use of needle knife papillotomy at $35 \%$, but this was evenly distributed between the groups. No statistical difference was found in the time to restenting or death with jaundice. 
Median patency for Tannenbaum stents was 181 (95\% CI 59 to 303) days and for Cotton-Leung stents 133 (92 to 174) days, and there was no statistical difference in the secondary end point of survival. The Kaplan-Meier analysis incorporated $42 \%$ of recruited patients as censored data, consisting of non-jaundiced patients who either died or withdrew from the study. Eight of 14 patients who remained jaundiced after first stenting died jaundiced without restenting. It is difficult to determine whether this poor uptake of restenting would have significantly affected the secondary end point of survival; five of these eight patients had received the Tannenbaum stent. Interestingly, a randomised prospective study comparing three patient groups who underwent either stent exchange for recurrent jaundice, three monthly prophylactic stent exchange or a single procedure with an expanding metal stent, showed no difference in overall survival in the three groups although the symptom-free survival was equally greater in the second two groups $(p<0.05) .{ }^{9}$ In the real world, however, the longer lasting stent is probably associated with increased survival, as was observed in a retrospective study, where the significantly increased survival in the metal stented group was associated with a lower percentage of patients dying with untreated occlusive jaundice $(9 \%)$ when compared with $30 \%$ in the plastic stent group ( $p<0.001){ }^{10}$

England et al's important work has shown that the more expensive Tannenbaum stent confers no significant advantages over the Cotton-Leung stent in the setting of a malignant biliary stricture where we believe, until adjuvant therapies significantly improve survival, a major role continues to exist for plastic stents. The relative performance of various designs in non-surgical candidates with a retained common duct stone merits further investigation. An independent group such as the Consumers Association, publishers of Which? magazine and the Drug and Therapeu- tics Bulletin, could become involved with evaluation of medical devices. Reviews of equipment used in many medical situations might be considered but the infrequency of reports in a given field would perhaps delay innovation. Manufacturers must grasp the nettle and justify claims for their products rather than passing responsibility for evaluation entirely to clinicians while reaping the commercial rewards.

M A NARAIN R COCKEL

University Hospital Birmingham,

Selly Oak Hospital,

Raddlebarn Road,

Birmingham B29 67D, UK

1 Medical Devices Agency. Directives Bulletins. London: Medical Devices Agency.

Seitz U, Vadeyar H, Soehendra N. Prolonged patency with a new-design Teflon biliary prosthesis. Endoscopy 1994;26:478-82.

3 Dowset JF, William SJ, Hatfield ARW, et al. Does stent diameter matter in the endoscopic palliation of malignant obstruction? A randomized trial of $10 \mathrm{~F}$ versus $12 \mathrm{~F}$ endoprostheses [abstract]. Gastroenterology 1989;96:A128.

4 Speer AG, Cotton PB, MacRae KD. Endoscopic management of malignant biliary obstruction: stents of 10 French gauge are preferable to stents of 8 French gauge. Gastrointest Endosc 1988;34:412-17.

5 Matsuda Y, Shimakura K, Akamatsu T. Factors affecting the patency of stents in malignant biliary obstructive disease: univariate and multivariate analysis. Am f Gastroenterol 1991;86:843-9.

6 Davids PHP, Groen AK, Rauws EAJ, et al. Randomised trial of self-expanding metal stents versus polyethylene stents for distal malignant self-expanding metal stents versus polyethylen

7 Knyrim K, Wagner HJ, Pausch J, et al. A prospective, randomised, controlled trial of metal stents for malignant obstruction of the common bile duct. Endoscopy 1993;25:207-12.

8 Lammer J, Hausegger KA, Flückiger F, et al. Common bile duct obstruction: treatment with plastic versus metal stents. Radiology 1996;201: $167-72$.

9 Prat F, Chapat O, Ducot B, et al. A randomized trial of endoscopic drainage methods for inoperable malignant strictures of the common bile duct. Gastrointest Endosc 1998;47:1-7.

10 Schmassmann A, von Gunten E, Knuchel J, et al. Wallstents versus plastic stents in malignant biliary obstruction: effects of stent patency of the first and second stent on patient compliance and survival. Am $\mathcal{f}$ Gastroenterol 1996;91:654-9.
Chronic infection with hepatitis $\mathrm{C}$ virus (HCV) affects $2-15 \%$ of the world's population. In the USA there are more than 4 million chronically HCV infected people and the estimates for Europe are similar. In the European Community chronic hepatitis $\mathrm{C}$ is viewed as a major health problem and efforts are being made to develop both preventive and new therapeutic strategies (Key Action 2 of the 5th Framework Programme 1998-2002 of Quality of Life and Management of Living Resources).

Treatment of chronic hepatitis C is currently based on interferon $\alpha(\mathrm{IFN}-\alpha)$ with limited efficacy, only achieving a sustained virological response in less than $25 \%$ of patients with a favourable profile treated for one year. ${ }^{1}$ To improve the response rate of standard IFN- $\alpha$ monotherapy, several regimens have been tried combining IFN- $\alpha$ three times weekly for six to 12 months with various other agents. These agents include those with antiviral activity like ribavirin, amantadine, ${ }^{2}$ and azidothymidine; others with immunomodulatory properties such as thymosin; those with liver enzyme lowering activity like ursodeoxycholic acid and ribavirin ${ }^{2}$; and others with miscellaneous properties such as ofloxacin, $\mathrm{N}$-acetylcysteine, glycyrrhizin, and non-steroidal anti-inflammatory drugs (NSAIDs). The aim of all these efforts was to increase IFN efficacy by decreasing the number of non-responders and relapsed responders as well as to reduce the dose of IFN- $\alpha$ required for a sustained response and consequently its side effects. The most rewarding of all these combinations with IFN was that of ribavirin which in large randomised controlled trials $^{34}$ proved to be far superior to IFN- $\alpha$ monotherapy of six and 12 months duration. In fact, the overall sustained response rate achieved with combination therapy in these two large trials reached $33 \%$ and $41 \%$ over six and 12 months of treatment, respectively, compared with $6 \%$ and $16 \%$ achieved with IFN monotherapy over the same time periods. The increase in sustained response was even more dramatic in patients with HCV genotypes 1 and 4-6 than in patients with the more easily eradicable genotypes 2 and 3. The sustained virological response doubled in the latter from $30 \%$ to $65 \%$ but tripled in the former from $9 \%$ to $30 \%$. However, despite these exciting results most treated patients with chronic hepatitis $\mathrm{C}$ fail to attain a sustained virological response. This is particularly true for infection with genotypes 1 and 4 , with $70 \%$ of infected patients failing to respond even to a 12 month combination therapy regimen. It is therefore conceivable that further efforts are needed in order to improve the efficacy of combination therapy. To this end different types of interferon-like consensus IFN, new formulations of IFN- $\alpha$ with attachment of polyethylene glycol, use of daily IFN dosing, higher doses and induction regimens aiming at rapid decrease of $\mathrm{HCV}$ replication are being tried. Combination of all these IFN 
directed efforts with adjuvant drugs is also under evaluation.

NSAIDs combined with IFN has been tried in naive and non-responsive patients with chronic hepatitis $\mathrm{C}$ with little or no success. ${ }^{5-7}$ However, existing evidence suggests that NSAIDs with their cyclooxygenase inhibiting activity possess an IFN potentiating effect by enhancement of 2',5'oligosynthetase activity. ${ }^{8}$ Negative results from the few early clinical studies of combined treatment with NSAIDs and IFN and case reports of NSAID induced hepatotoxicity ${ }^{9}$ in chronic hepatitis have been discouraging.

In this issue (see page 427), Muñoz et al report on a prospective randomised controlled trial evaluating the efficacy of combined treatment with IFN and ketoprofen versus IFN alone. The authors have managed to study a fairly large, for a single centre, number of patients with well defined inclusion criteria of chronic hepatitis C. The patients were allocated into three treatment groups receiving either: (1) IFN alone at 3 million units three times weekly for six months; (2) IFN combined with a small dose of the NSAID ketoprofen, $200 \mathrm{mg}$ given three hours before the thrice weekly injections of IFN; or (3) IFN combined with daily ketoprofen at a dose of $200 \mathrm{mg}$ given twice a day. Ketoprofen was administered as a slow-release form. Both groups also received ranitidine $(150 \mathrm{mg} /$ day $)$ prophylactically. Ketoprofen was selected over other NSAIDs because it has been used in the past in patients with hepatitis without serious side effects, it has a high bioavailability, its pharmacokinetics are not influenced by cirrhosis, and it inhibits both the cyclooxygenase and lipooxygenase inflammatory pathways. The patients were monitored carefully clinically, biochemically, and virologically. $\mathrm{HCV}$ RNA was measured by RT-PCR and negative cases were retested by a nested PCR with a detection limit of 1000 copies $/ \mathrm{ml}$. Both doses of ketoprofen were well tolerated with similar side effects to the IFN monotherapy arm except perhaps for a greater drop in haemoglobin concentrations. The virological response rate at the end of follow up (sustained virological response) was significantly higher in the daily ketoprofen group (26\%) compared with the small thrice weekly dose and to the IFN monotherapy groups $(0 \%$ and $10 \%$, respectively, and $2 \%$ in the two groups combined, $p=0.009)$. The response rate of the few cirrhotic patients in group 3 was similar. Moreover the 'flu-like side effects of IFN- $\alpha$ were less frequent in the ketoprofen treated patients.

This is the first time that ketoprofen has been given to patients with chronic hepatitis $C$ twice daily in a slow-release form, similar to the doses recommended for the treatment of osteoarthritis and rheumatoid arthritis. This probably explains the major difference in the efficacy of this regimen compared with the lower dose scheme and with the negative results of a similar study using a different ketoprofen dosage. ${ }^{6}$ Independently of these encouraging results, there are some points of concern in this study that have also been recognised by the authors. Firstly, as expected, the number of patients studied, although large for a single centre study $(\mathrm{n}=70)$, is still very small for a three arm study and both type 1 and type 2 errors are possible. In fact the difference between groups 1 and 3 is not statistically significant. Furthermore, because of the small number of patients an intent-to-treat analysis weakens the differences significantly. Secondly, it is difficult to explain the very large discrepancy between the rate of biochemical and virological response at the end of treatment observed in all three groups. Even in the IFN monotherapy group the biochemical response rate was $52 \%$ and the virological rate $10 \%$. Thirdly, it is not conceivable how the group on the small ketoprofen dose could have had a response rate below that of the IFN monotherapy group. Fourthly, again as expected, owing to the small number of patients studied, the effect on the efficacy of therapy of several baseline variables such as viral load, HCV genotypes, and extent of liver fibrosis was not evaluated. These and other emerging questions should be answered in future trials of large numbers of patients.

Regardless of the study's limitations, this report of a significant increase in the sustained virological response rate achieved at a small additional financial cost should herald new clinical trials of combined treatment with ketoprofen and IFN. The postulate that NSAIDs may potentiate the action of IFN has received timely independent support from a recent study in hepatoma cell lines, indicating that blockade of arachidonic acid metabolism by NSAIDs, activates a signalling pathway potentiating IFN- $\alpha$ dependent gene activation. ${ }^{10}$ Clearly the last word on the combination of NSAIDs with IFN has still to be written.

Academic Department of Medicine,

S J HADZIYANNIS

Hippokration General Hospital,

114 Vas. Sophias Avenue,

11527 Athens, Greece

1 EASL International Consensus Conference on Hepatitis C. Consensus Statement. European Association for the Study of the Liver. $\mathcal{F}$ Hepatol 1999;30:956-61.

2 Younossi ZM, Perrillo RP. The roles of amantadine, rimantadine, ursodeoxycholic acid, and NSAIDs, alone or in combination with alpha interferons, in the treatment of chronic hepatitis C. Semin Liver Dis 1999; 19(suppl 1):95-102.

3 Poynard T, Marcellin P, Lee SS, et al. Randomised trial of interferon alpha2b plus ribavirin for 48 weeks or for 24 weeks versus interferon alpha $2 \mathrm{~b}$ plus placebo for 48 weeks for treatment of chronic infection with hepatitis C cet 1998;352:1426-32.

4 McHutchison JG, Gordon SC, Schiff ER, et al. Interferon alfa-2b alone or in combination with ribavirin as initial treatment for chronic hepatitis C. Hepatitis Interventional Therapy Group. N Engl f Med 1998;339:1485-92.

5 Andreone P, Cursaro C, Gramenzi A, et al. Alfa-Interferon plus indomethAndreone $\mathrm{P}$, Cursaro $\mathrm{C}$, Gramenzi A, et al. Alfa-Interferon plus indometh-
acin combined therapy for non-responder patients with chronic hepatitis $\mathrm{C}$ acin combined therapy for non-respon
[abstract]. Hepatology 1993;18:A221.

6 Anderson FH, Zeng L, Yoshida EM, et al. Failure of ketoprofen and interferon combination therapy to improve interferon-resistant chronic hepatitis C. Can $\mathcal{F}$ Gastroenterol 1997;11:294-7.

7 Zarski JP, Maynard-Muet M, Chousterman S, et al. Tenoxicam, a non-steroid anti-inflammatory drug, is unable to increase the response rate in patients with chronic hepatitis $\mathrm{C}$ treated by alpha interferon. Hepatology 1998;27:862-7.

8 Andreone P, Cursaro C, Gramenzi A, et al. Indomethacin enhances serum 2 '5'-oligoadenylate synthetase in patients with hepatitis $B$ and $C$ virus chronic active hepatitis. F Hepatol 1994;21:984-8.

9 Riley TR, Smith JP. Ibuprofen-induced hepatotoxicity in patients with chronic hepatitis C: a case series. Am f Gastroenterol 1998;93:1563-5.

10 Giambartolomei S, Artini M, Almerighi C, et al. Nonsteroidal antiinflammatory drug metabolism potentiates interferon alfa signaling by increasing STAT1 phosphorylation. Hepatology 1999;30:510-16. 